\title{
Multiple thoracic disc herniations: case report and review of the literature
}

\author{
Y Okada $^{1}$, K Shimizu ${ }^{1}, \mathrm{~K} \mathrm{Ido}^{1}$ and S Kotani ${ }^{2}$ \\ ${ }^{1}$ Department of Orthopaedic Surgery, Faculty of Medicine, Kyoto University, 54 Shogoin Kawaharacho, Sakyoku, \\ Kyoto 606, Japan; ${ }^{2}$ Department of Orthopaedic Surgery, Maizuru Municipal Hospital, Japan
}

\begin{abstract}
The incidence of intervertebral disc herniation in the thoracic region of the spine is much less than in the cervical or lumbar areas, and multiple thoracic disc herniations are rare. We described a 33-year-old man with two-level thoracic disc herniation, who exhibited features of spinal cord compression. Magnetic resonance imaging and computed tomographic myelography demonstrated anterior compression of the spinal cord due to disc herniation at T4/5 and T7/8 levels. Through an anterolateral approach, these discs were removed and interbody fusion was performed using autogenous bone grafts. Excellent results were obtained.
\end{abstract}

Keywords: anterolateral approach; multiple disc herniations; spinal cord compression; thoracic spine

\section{Introduction}

Thoracic disc herniation is relatively uncommon, and multiple thoracic disc herniations are rare. However, the identification of thoracic disc herniation has increased recently. We present a patient with thoracic disc herniations at two levels, and discuss the diagnosis of this condition and its surgical treatment.

\section{Case report}

A 33-year-old male factory worker noticed numbness in both lower extremities in July 1993. There was no history of trauma and he had no back pain. Weakness of his lower extremities and a spastic gait had appeared by August 1994. On examination, there was slight muscle weakness and exaggerated tendon reflexes in both lower extremities, bilateral patellar and ankle clonus, a spastic gait and sensory disturbances in the lower legs. However, there was no muscle atrophy or bladder or bowel dysfunction. Blood, biochemical, and urine analyses revealed no abnormalities.

Plain radiography showed slight scoliosis, but no other abnormalities. Magnetic resonance imaging (MRI) demonstrated anterior compression of the spinal cord due to disc herniation at $\mathrm{T} 4 / 5$ and $\mathrm{T} 7 / 8$ (Figure 1). Myelography showed small anterior extradural defects at $\mathrm{T} 4 / 5$ and $\mathrm{T} 7 / 8$ (Figure 2). Computed tomographic (CT) myelography also showed anterior compression of the spinal cord due to disc herniation at $\mathrm{T} 4 / 5$ and $\mathrm{T} 7 / 8$ (Figure 3 ). The patient underwent surgery through an anterolateral transthoracic approach on 26 October, 1994. The discs

Correspondence: Yoshifumi Okada at $\mathrm{T} 4 / 5$ and $\mathrm{T} 7 / 8$ were removed under a surgical microscope and intervertebral fusion was performed using a resected fourth rib. The postoperative course was uneventful. Postoperative CT-myelography showed complete removal of the herniated discs (Figure 4). Twenty two months after the operation, the patient showed full recovery of neurological functions except for minor numbness in the lower legs and bone fusion was confirmed by radiography.

\section{Discussion}

Thoracic disc herniation is an unusual condition, and accounts for only $0.15 \%$ to $1.8 \%$ of all intervertebral disc abnormalities treated surgically. ${ }^{1-3}$ It affects the sexes equally, and is more common between the fourth and sixth decades with a peak in the fourth decade. ${ }^{2,3}$ Although thoracic disc herniations have been reported at every level, $75 \%$ occur below T8 with a peak at T11/ 12 , where there is greater spinal mobility. ${ }^{1,4,5}$ Weakness of the posterior longitudinal ligament in the lower thoracic spine is also a causative factor. ${ }^{6}$ Such herniation can be divided into central, centrolateral, or lateral types. Central protrusions are the most common. ${ }^{1}$ Some series have implicated trauma as a cause of thoracic disc herniation, ${ }^{5}$ but in our patient there was no history of trauma. In thoracic disc herniation, the gradual onset of clinical manifestations can lead to a delay in diagnosis. ${ }^{2}$ Multiple thoracic disc herniations are rare; the condition was first reported by Svien and Karavits in $1954 .^{7}$ However, the identification of multiple thoracic disc herniations has increased because of the recent introduction of MRI. Wood et al. reviewed MRI studies of the thoracic spines of 90 
asymptomatic volunteers. At least one disc was herniated in $33(37 \%)$ of the 90 volunteers and 13 $(39 \%)$ of the 33 individuals who had at least one herniation had multiple herniations. ${ }^{8}$ But, symptomatic multiple thoracic disc herniations treated surgically are very rare.

a

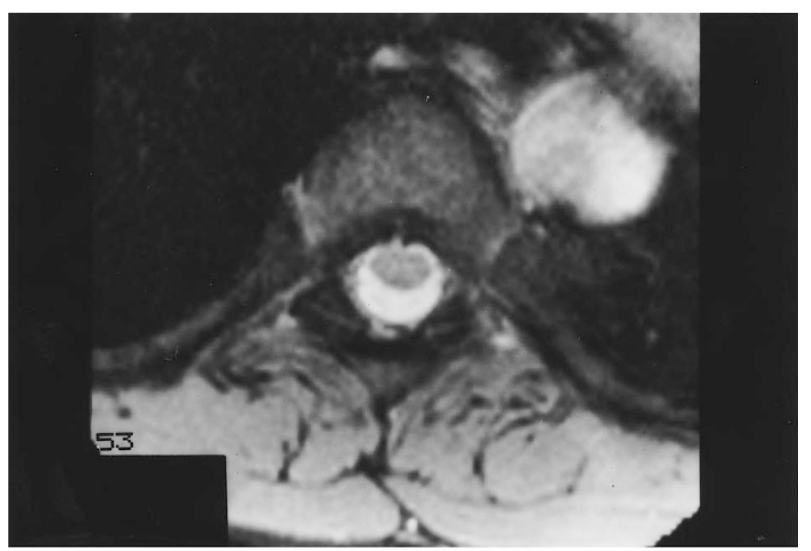

b

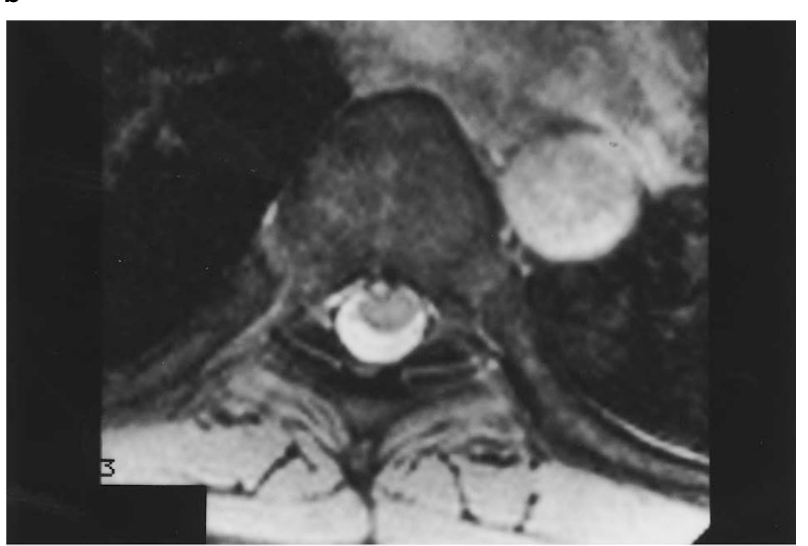

C

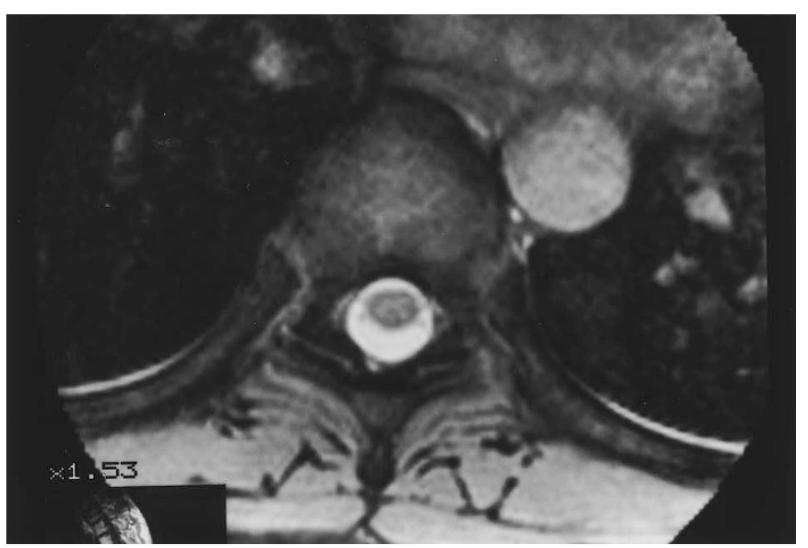

Figure 1 (a) Axial MRI at T4/5 shows small herniated disc. (b) Axial MRI at T7/8 shows herniated disc compressing the anterior surface of the spinal cord. (c) Normal thoracic level for comparison. (T8/9)
Radiography of the thoracic spine may reveal narrowing of the intervertebral disc space, osteophyte formation, narrowing or sclerosis of the apophysial joint and calcification within the intervertebral disc. The first three findings are of little value for the diagnosis of thoracic disc herniation. Calcification within the intervertebral disc is a helpful indicator, 7,9 but this was not evident in our patient. Some authors have reported that the incidence of disc calcification in the radiography of protruded thoracic discs ranges between $70 \%$ and $75 \%$ compared with $4 \%$ for normal patients. ${ }^{9,10}$ Myelography is useful for the diagnosis of thoracic disc herniation, ${ }^{10}$ although there may be some false negative results. CT-myelography is diagnostically useful, ${ }^{5,11}$ and Alvarez considered this to be superior to all other imaging modalities demonstrating in detail the degree of encroachment on the subarachnoid space, the degree of spinal cord compression and the integrity of the bony architecture of the spinal canal at the affected levels. ${ }^{11}$ Coleman stated that CT-myelography was a useful surgical guide to a laterally placed lesion, and allowed assessment of any abnormality of the posterior

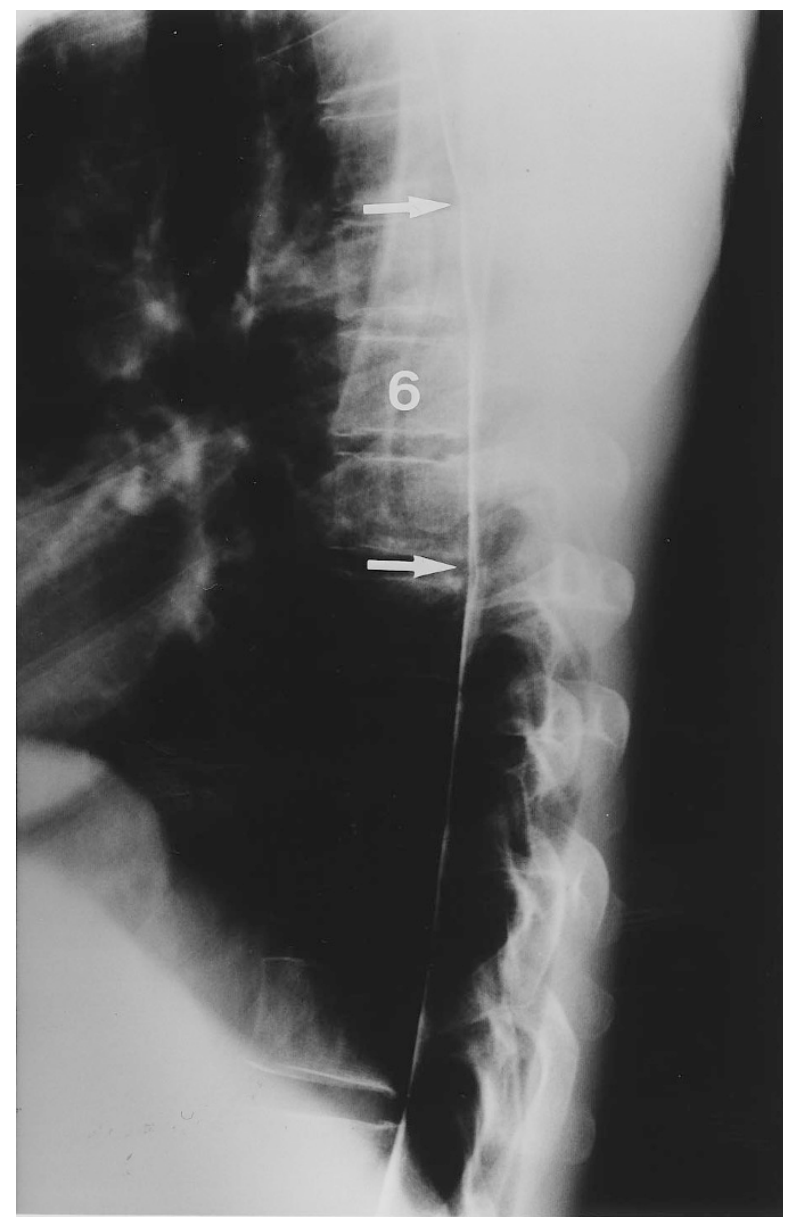

Figure 2 Lateral myelography shows small anterior extradural defects at $\mathrm{T} 4 / 5$ and $\mathrm{T} 7 / 8$ 
structures which may accompany disc prolapse in patients with vertebral canal stenosis. ${ }^{12}$ In our patient, CT-myelography showed thoracic disc herniations at the T4/5 and T7/8 levels. Recently, MRI has also become an effective method for the diagnosis of

\section{a}

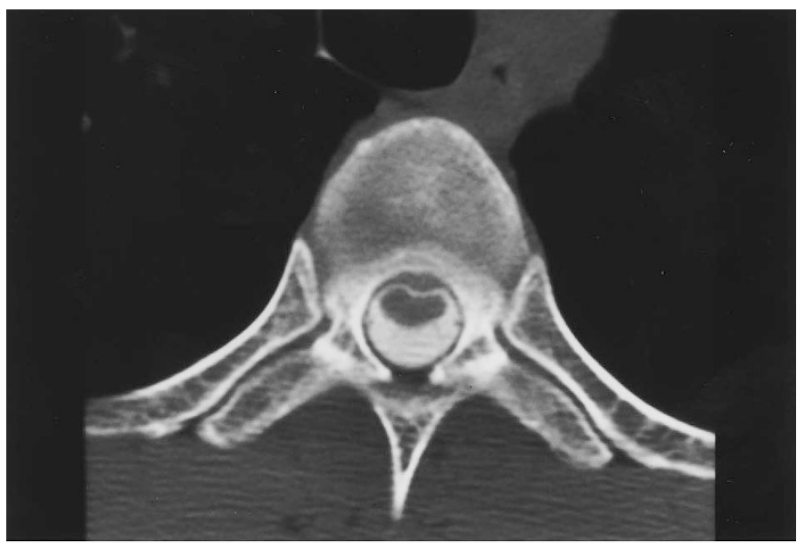

b

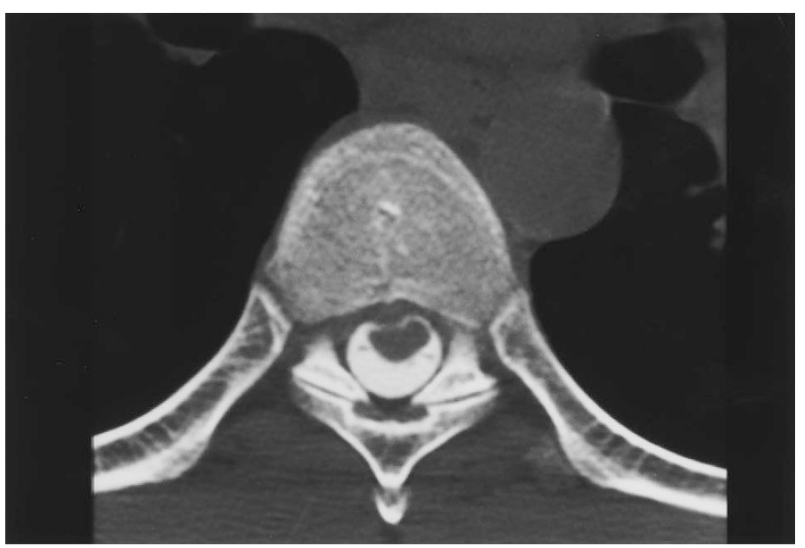

C

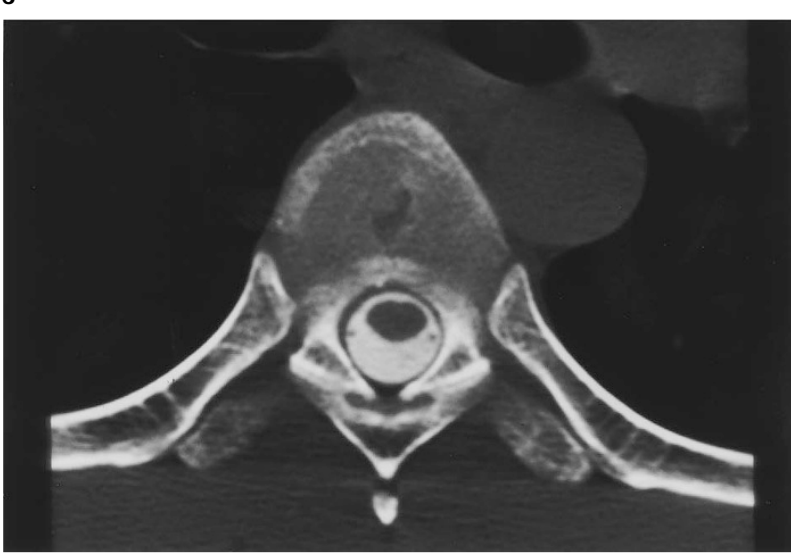

Figure 3 (a) CT-myelography at T4/5 shows herniated disc compressing the anterior surface of the spinal cord. (b) CTmyelography at $\mathrm{T} 7 / 8$ shows findings similar to those in a. (c) Normal thoracic level for comparison. (T6/7)

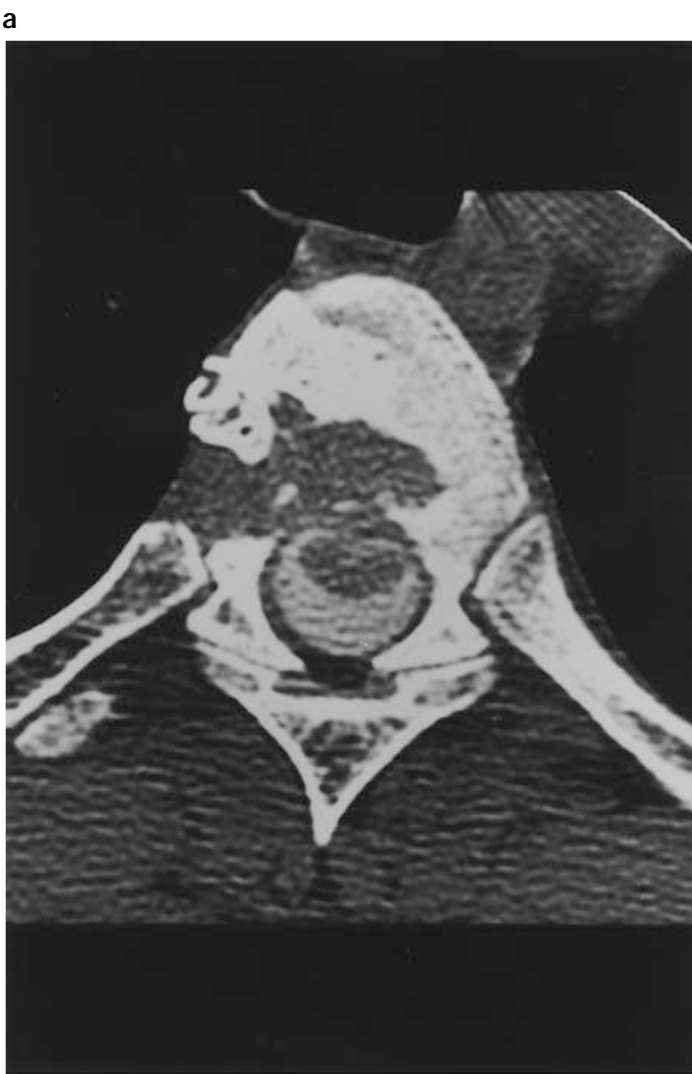

b

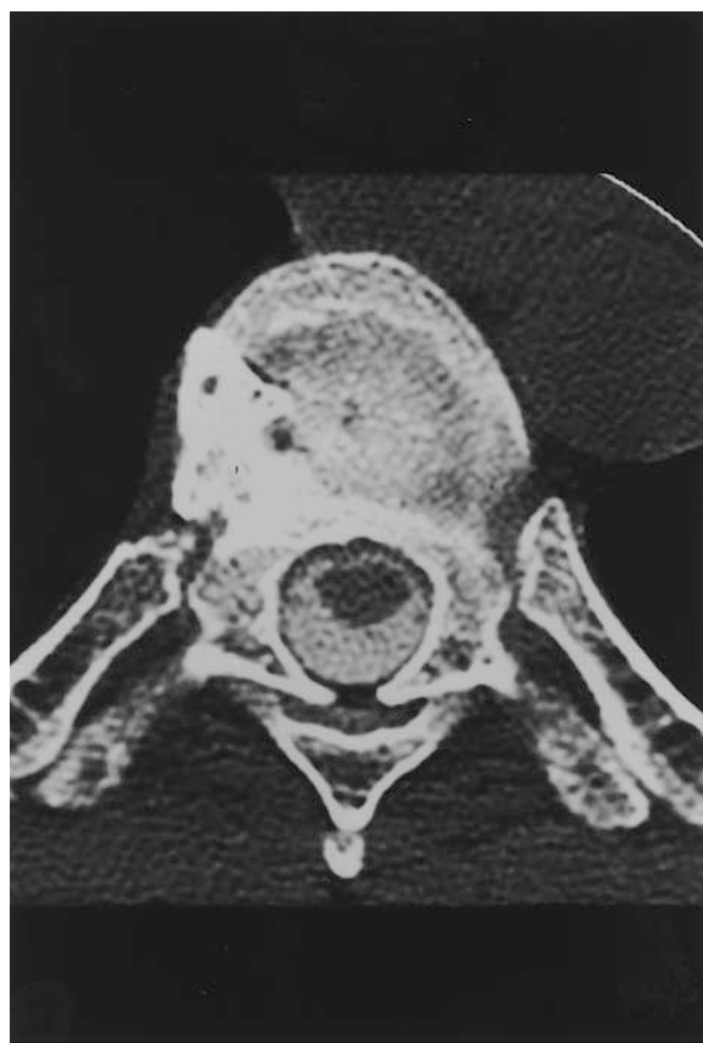

Figure 4 Postoperative CT-myelography at T4/5 (a) and at 
thoracic disc herniation ${ }^{13}$ and because it lacks the attendant risks of ionizing radiation, it may render myelography obsolete. ${ }^{11}$

Conservative treatment is not effective, and surgery alone is considered to give satisfactory results for patients with thoracic disc herniations. Symptomatic patients must be treated as early as possible, because the thoracic spinal canal is narrow and allows little infringement of the subarachnoid space. ${ }^{13}$ Generally, it is difficult to determine which of the herniated thoracic discs causes the symptom by neurological findings only. Moreover, reoperation is difficult in the thoracic region. Thus, we performed operation for the two-level disc herniations. Laminectomy has been performed for thoracic disc herniation, but the results were poor. ${ }^{2,9,14}$ A compressive lesion which lies anterior to the spinal cord is commonly median in position and thus only poorly accessible by the posterior approach. The spinal cord at the site of compression may be far more vulnerable to damage from relatively minor trauma because of its poor vascularity. Laminectomy may produce an abnormal increase in spinal motion because it removes the posterior structures of the spinal column, and therefore the results may be poor. ${ }^{2}$ Crafoord et al. reported the first use of the transthoracic approach for removal of the disc in a patient with thoracic disc herniation in $1958,{ }^{15}$ and this approach was subsequently developed by Perot and Munro ${ }^{16}$ and Ransohoff et al. ${ }^{17}$ This technique offers the best opportunity for removal of the compressive lesion with the least manipulation of the compromised spinal cord ${ }^{17}$ and allows both hard and soft discs to be removed easily. ${ }^{13}$ Thus anterior surface of the dural sac is also better visualised. ${ }^{13}$ Patterson and Arbit reported the extrapleural transpedicular approach, ${ }^{18}$ which was later modified by Carson et al. ${ }^{19}$ This approach is much better for soft discs than for calcified discs or osteophytes. ${ }^{14}$ Accurate preoperative diagnosis is essential. The preferred approach is either anterolateral transthoracic or posterolateral transpedicular. ${ }^{2,14,15,18}$ In our patient, surgery was performed through an anterolateral transthoracic approach, and excellent results were obtained.

\section{Acknowledgements}

We thank Noboru Nakayama, MD, Department of Surgery, Maizuru Municipal Hospital, for operative assistance and Makoto Yoshida, MD, Department of Orthopaedic Surgery, Maizuru Municipal Hospital, for clinical assistance.

\section{References}

1 Love JG, Schorn VG. Thoracic-disk protrusions. JAMA 1965; 191: $627-631$

2 Otani K et al. Surgical treatment of thoracic disc herniation using the anterior approach. J Bone Joint Surg 1982; 64B: $340-343$.

3 Bhole R, Gilmer RE. Two-level thoracic disc herniation. Clin Orthop 1984; 190: $129-131$.

4 Haley JC, Perry JH. Protrusions of intervertebral discs: Study of their distribution, characteristics and effects on the nervous system. Am J Surg 1950; 80: 394-404.

5 Arce CA, Dohrmann GJ. Thoracic disc herniation: Improved diagnosis with computed tomographic scanning and a review of the literature. Surg Neurol 1985; 23: 356-361.

6 Peker S, Akkurt C, Ozcan OE. Multiple thoracic disc herniations. Acta Neurochir 1990; 107: $167-170$.

7 Svien HJ, Karavitis AL. Multiple protrusions of intervertebral disks in the upper thoracic region: Report of case. Staff Meet Mayo Clin 1954; 29: 375-378.

8 Wood KB et al. Magnetic Resonance Imaging of the Thoracic Spine. J Bone Joint Surg 1995; 77A: $1631-1638$.

9 Logue V. Thoracic intervertebral disc prolapse with spinal cord compression. J Neurol Neurosurg Psychiatry 1952; 15: $227-241$

10 McAllister VL, Sage MR. The radiology of thoracic disc protrusion. Clin Radiol 1976; 27: $291-299$.

11 Alvarez O, Roque CT, Pampati M. Multilevel thoracic disk herniations: CT and MR studies. J Comput Assist Tomogr 1988; 12: $649-652$.

12 Coleman RJ, Hamlyn PJ, Butler P. Anterior spinal surgery for multiple thoracic disc herniations. British Journal of Neurosurgery 1990; 4: $541-543$.

13 Shikata J et al. Multiple thoracic disc herniations: Case report. Neurosurgery 1988; 22: $1068-1070$.

14 Sekhar LN, Jannetta PJ. Thoracic disc herniation: Operative approaches and results. Neurosurgery 1983; 12: $303-305$.

15 Crafoord TC et al. Spinal cord compression caused by a protruded thoracic disc: Report of a case treated with anterolateral fenestration of the disc. Acta Orthop Scand 1958; 28: $103-107$.

16 Perot PL Jr, Munro DD. Transthoracic removal of midline thoracic disc protrusions causing spinal cord compression. $J$ Neurosurg 1969; 31: $452-458$.

17 Ransohoff $\mathbf{J}$ et al. Transthoracic removal of thoracic disc: Report of three cases. J Neurosurg 1969; 31: 459-461.

18 Patterson RH Jr, Arbit E. A surgical approach through the pedicle to protruded thoracic discs. J Neurosurg 1978; 48: $768-$ 772

19 Carson J, Gumpert J, Jefferson A. Diagnosis and treatment of thoracic intervertebral disc protrusions. J Neurol Neurosurg Psychiatry 1971; 34: 68-77. 